Classification

Physics Abstracts

$61.30 \mathrm{E}-64.70 \mathrm{M}-77.80 \mathrm{D}$

\title{
Smectic $X$ - the first truly ferroelectric liquid crystal
}

\author{
H. R. Brand (*) and P. E. Cladis \\ Bell Laboratories, Murray Hill, New Jersey 07974, U.S.A.
}

(Reçu le 15 novembre 1983, accepté le 17 janvier 1984)

\begin{abstract}
Résumé. - Nous présentons des résultats expérimentaux permettant d'identifier la première mésophase véritablement ferroélectrique dans un composé qui présente également des phases $C$, I et $G^{\prime}$ à plus haute température. Dans cette nouvelle phase, le directeur s'oriente de façon bistable sous un champ électrique $V$. Le temps caractéristique $\tau$ de ce processus est tel que $V \cdot \ln \tau$ est proportionnel à la température. De plus, l'échantillon reste orienté même après la suppression du champ. Ce comportement indique une différence fondamentale entre cette nouvelle phase et les smectiques chiraux en volume.
\end{abstract}

\begin{abstract}
We report the identification of the first truly ferroelectric liquid crystal in a compound which also shows chiral $\mathrm{C}$, I and $\mathrm{G}^{\prime}$ phases at higher temperatures. In the new phase the director can be oriented in a bistable way with an electric field $V$ in a time $\tau$ such that $V \cdot \ln \tau$ is proportional to temperature. Furthermore, when the field is turned off, the orientation remains. This behaviour indicates a fundamental difference between the new phase and bulk chiral smectics.
\end{abstract}

In recent years there has been increasing interest in the stable phases mediating the transition between the solid and the liquid phase in many organic compounds. In particular, interest has focussed on the layered phases known as smectic liquid crystals [1] in which the degree of order within the layers changes quite often in a very subtle way from one phase to another. In smectic $\mathrm{C}$, for example, the director describing the preferred direction along which the long axis of the rod like molecules tends to align is tilted relative to the layer normal. As in the nontilted smectic A phase, there are only short-range positional correlations within the layers. Upon cooling, a smectic $\mathrm{C}$ phase may be followed by a smectic $\mathrm{I}\left(\right.$ or $\mathrm{F}^{2}$ ) phase $[3,4]$, in which long-range bondorientational order is believed to set in [4] whereas the positional correlations remain shortrange. A further reduction of temperature leads to the onset of long-range positional order in the smectic $\mathrm{G}\left(\mathrm{G}^{\prime}\right)$ and $\mathrm{H}\left(\mathrm{H}^{\prime}\right)$ phases [4]. These phases are considered to be crystals on the basis of sharp Bragg spots in X-ray diffraction [4] and of a finite shear modulus inside the layers [5]. The molecular disorder in these phases, however, is still very large $[3,6]$ and not all the rotational degrees of freedom have been frozen out $[3,6]$.

The purpose of this paper is to point out that in some of the " crystalline » liquid crystal phases of chiral molecules, the director orientation can be changed in a bistable manner with a dc electric field. The existence of ferroelectricity is well known in the solid state but its existence has not been

$\left(^{*}\right)$ Present address : Dept. of Physics, Kyushu University, Fukuoka 812, Japan. 
predicted in the liquid crystalline state where, unlike in the solid state, the application of an electric field stimulates a physical reorientation of the molecules.

Although chirality is a molecular property, in the literature, chiral smectics refers to those liquid crystalline phases (sometimes formed by chiral molecules) in which there is both a layered structure in which the molecules are tilted relative to the layer normal and a helicoidal structure which results when the tilt direction rotates about an axis perpendicular to the layers with a pitch much larger than the layer thickness. The spontaneous polarization resulting from the combination of molecular chirality, tilt and layers $[1,7,8]$ is perpendicular to the layer normal. Since it is rigidly coupled to the director, it globally averages to zero when a helicoidal structure is present. Thus, all ferroelectric liquid crystals known to date, $\mathrm{C}^{*}, \mathrm{~F}^{*}, \mathrm{I}^{*}[7]$, with the asterisk indicating the spiral structure of these phases, are not ferroelectric in the usual sense [1]. We believe smectic $X$ to be the first truly ferroelectric liquid crystal.

We do not know the in-plane structure of X. It may be similar to, for example, a non-helicoidal $\mathrm{G}$ or $\mathrm{H}^{\prime}$ phase, two phases which also occur at lower temperatures than the $\mathrm{G}^{\prime}$ phase. To date, these phases have not been shown to be ferroelectric even when they are composed of chiral molecules. Since a spontaneous polarization is the important feature of $\mathrm{X}$, should it share the crystallographic structure of $G$, say, then we propose it be called $\hat{G}$ to set it apart from a $G$ phase which is not ferroelectric. Since the helicoidal structure is absent, $G^{*}$ would be misleading. We have found $X$ in a variety of compounds. This means that it is not likely to be the property of a unique in-plane structure.

The material we studied is called $8 \mathrm{SI}^{*} \Delta \mathrm{S}-(+)-\left(4-\left(2^{\prime}\right.\right.$-methylbutyl) phenyl 4'-n-octylbiphenyl-4carboxylate). In this compound we observed the following sequence of transitions :

$$
\mathrm{Cr} \underset{40.9}{\stackrel{48.2}{\rightleftarrows}} \mathrm{X} \stackrel{61.7}{\rightleftarrows} \mathrm{G}^{\prime *} \stackrel{66.5}{\rightleftarrows} \mathrm{I}^{*} \stackrel{71.1}{\rightleftarrows} \mathrm{C}^{*} \stackrel{81}{\rightleftarrows} \mathrm{A} \stackrel{137.2}{\rightleftarrows} \mathrm{Chol} . \stackrel{138.7}{\rightleftarrows} \mathrm{BP} \stackrel{140}{\rightleftarrows} \mathrm{Isotr}^{\circ}{ }^{\circ} \text {. }
$$

Except for the blue phases, we found an exact correspondence between the sequence of phases exhibited by 8SI* and its racemate 8SI. The temperatures of the phase transitions have been determined by microscopic observations and DSC measurements. There is a large heat of transition $(1.12 \mathrm{cal} / \mathrm{g})$ associated with $\mathrm{I}^{*} \rightleftarrows \mathrm{C}^{*}$ whereas the texture change is very small. The heat of transition for $X \rightleftarrows G^{*}$ is very small (less than $0.01 \mathrm{cal} / \mathrm{g}$ ) and we could not detect the transition $\mathrm{G}^{* *} \rightleftarrows \mathrm{I}^{*}$ in DSC which indicates that this transition is (nearly) of second order. On cooling through $\mathrm{X}$, the transition to a crystal phase takes place at $40.2^{\circ} \mathrm{C}$ with a heat of transition of $1.8 \mathrm{cal} / \mathrm{g}$. This is considerably less than $8.5 \mathrm{cal} / \mathrm{g}$ observed at the melting transition $\left(48.2^{\circ} \mathrm{C}\right)$ but larger than any of the heats of transitions at the higher temperature transitions. In this sense then, we consider all phases of $8 \mathrm{SI}^{*}$ above $40^{\circ} \mathrm{C}$ "liquid crystalline » as opposed to simply « crystalline ».

Chiral smectic phases are optically uniaxial, because of the helix structure, whereas the $\mathrm{X}$ phase turned out to be biaxial. To observe this we looked at a free standing film of 8SI* in the microscope. All our films were drawn in the smectic A phase $\left(100^{\circ} \mathrm{C}\right)$ and showed the same degree of optical extinction in the $\mathrm{C}^{*}, \mathrm{I}^{*}$ and $\mathrm{G}^{*}$ phase between crossed polarizers. Only changes in fluidity marked these transitions upon heating and cooling. The transition $\mathrm{G}^{*} \rightleftarrows \mathrm{X}$ not only shows a change in fluidity but also the sudden appearance of birefringence colours just below $62{ }^{\circ} \mathrm{C}$ and the film lost its smoothness. From this we infer the biaxial nature of this phase. We distinguish between the colours of optical rotatory power and biaxiality by rotating the film between crossed polarizers. In the former case there is no change in colour nor in intensity of transmitted light whereas in the latter there is. Thus $\mathbf{X}$ is biaxial in our films.

We cannot exclude the possibility that the pitch jumps from about $3 \mu$, its value in $\mathrm{G}^{*}$, to some value greater than the film thickness but still finite, in X. Presumably, as long as this number is much greater than a sample thickness, $X$ will still be effectively ferroelectric. Assuming a flexoelectric coupling [1] between polarization, $\mathbf{P}$, and a deformation like curl $\mathbf{n}$ where $\mathbf{n}$ is the director, 
a standard calculation estimates the expected pitch to be on the order of 1-2 $\mathrm{mm}$ in the $\mathrm{C}^{*}$ phase where the $|\mathbf{P}| / K, K$ being a Frank constant, may be orders of magnitude larger than it is in $\mathbf{X}$. Thus in $\mathrm{X}$, the pitch, if there is one, would be even larger, so, effectively infinite.

The most elegant and clearcut way of distinguishing the $I^{*}, G^{*}$ and $C^{*}$ phases on the one hand and the $\mathrm{X}$ phase on the other is certainly the different electrooptic behaviour of these phases. In $8 \mathrm{SI}^{*}, \mathrm{X}$ possesses a net permanent polarization (whereas its counterpart in $8 \mathrm{SI}$ does not) and can be switched in a bistable way in an electric field. Unlike $I^{*}, \mathrm{G}^{*}$ and $\mathrm{C}^{*}$, however, the orientation of the director does not change in the $\mathrm{X}$ phase when the field is turned off.

The existence of a permanent polarization in smectic $X$ was demonstrated by applying a dc electric field across a $12 \mu \mathrm{m}$ thick sample (see Fig. 1). In order to obtain the well-ordered sample depicted in figures 1 and 2, the sample of 8SI* was rapidly cooled from the isotropic phase to the A phase in a magnetic field of $10 \mathrm{kG}$ parallel to the electrode surface. This technique with slow cooling gives well-ordered smectic phases of relatively thick samples $(\sim 50 \mu)$ provided there is a smectic A-isotropic transition. Cooling 8SI* rapidly enabled us to "short-circuit » the narrow cholesteric phase (and blue phases) and smectic A batonnets were formed in the isotropic melt. These coalesced to form monodomains on the order of $400-500 \mu \mathrm{m}$ (see Fig. 2) in the usual way [10]. We did not treat the glass plates with any surfactant.

At $66^{\circ} \mathrm{C}$, we observed in the polarizing microscope that $15 \mathrm{~V}$ cleared the sample of the surface lines shown in the top of figure 2 . When the field was turned off, the director configuration relaxed to the helicoidal structure (in seconds or less) [12] and surface lines reappeared in about a minute. Figure 2 shows the state of the sample at this point when the surface lines are about $4.6 \mu \mathrm{m}$ apart.

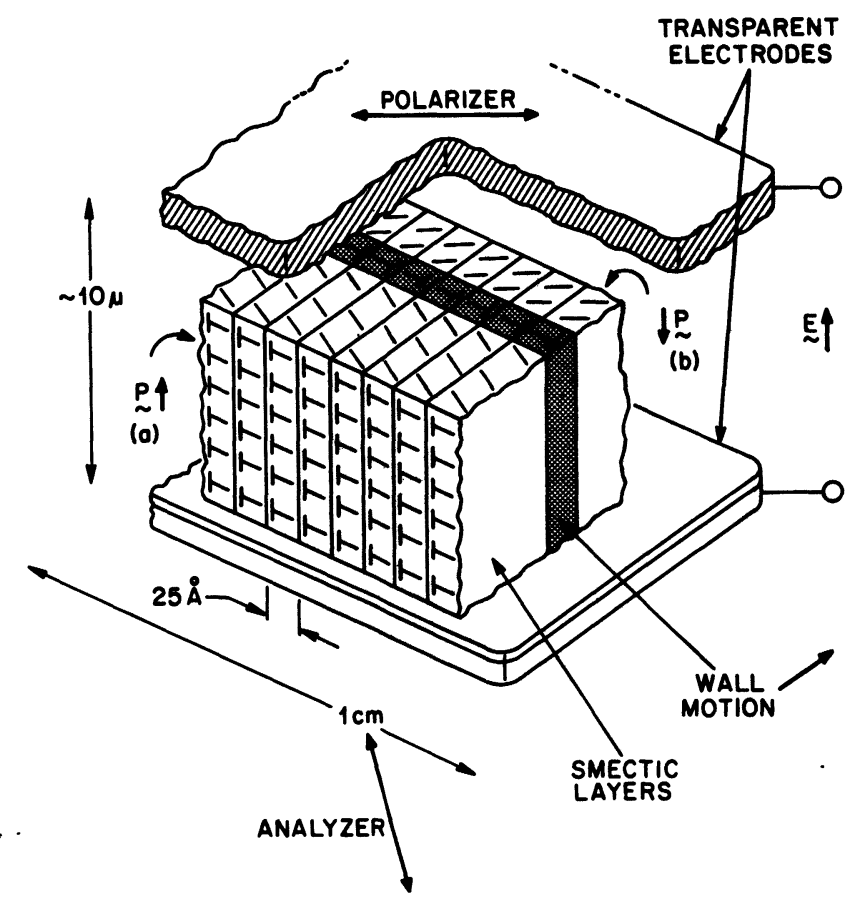

Fig. 1. - Schematic of the two states of well-ordered smectic $X$ phase, which occurs at lower temperatures than the $\mathrm{G}^{\prime *}$ liquid crystal phase which has been characterized as a crystal. We do not know the detailed in-plane structure of X.P is the polarization which is « up » for one state (a) and « down " for the other (b). In an applied field, $\mathrm{X}$ switches from state (a) to (b) via wall motion parallel to the layers. The nail-head indicates the end of the director behind the page. 


\section{$8 \mathrm{SI}^{*}$}
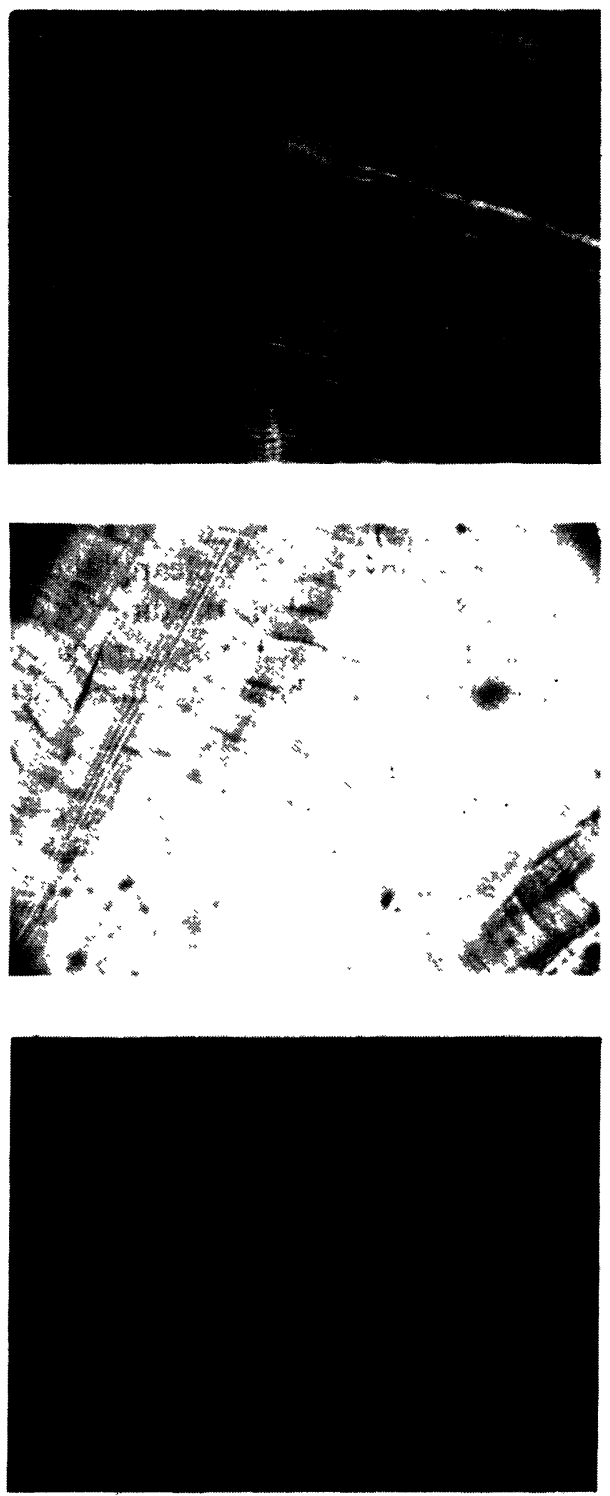

$66^{\circ} \mathrm{C}$

$61^{\circ} \mathrm{C}$

(a)

$61^{\circ} \mathrm{C}$

(b)

Fig. 2. - Optical micrographs of $8 \mathrm{SI}^{*}$ at $66^{\circ} \mathrm{C}$ and $61^{\circ} \mathrm{C}$ for states (a) and (b). The sample is viewed through the transparent electrodes of figure 1.

With time, more lines appear. Cooling in steps of $1{ }^{\circ} \mathrm{C}$ to $62^{\circ} \mathrm{C}$, we repeated this experiment and found qualitatively the same behaviour : the helicoidal structure reappeared (in seconds or less) and the surface lines grew back (in 1 or $2 \mathrm{~min}$.). At $61^{\circ} \mathrm{C}$ something different happened. The director re-oriented slowly $(\sim \mathrm{s})$ in a $12 \mathrm{~V}$ dc field but no change was observed at the end of an hour after the field had been switched off(state (a) in Fig. 2). When the sign of the field was reversed, 
the director reoriented to state (b) in figure 2 and again, when the field was switched off, no change occurred.

Unlike the phases above $61^{\circ} \mathrm{C}$, there was now a threshold voltage, $V_{\mathrm{C}}$, to reorient the director. For example, at $58^{\circ} \mathrm{C}$, no change was observed for fields less than $50 \mathrm{~V} / 12 \mu$. In the vicinity of $V_{\mathrm{C}},\left(\left(V-V_{\mathrm{C}}\right) / V_{\mathrm{C}} \simeq 50 \%\right)$, we measured with a stop-watch the times to change from state (a) to state (b) across a fixed area of the sample (about $500 \mu \mathrm{m}^{2}$ ) somewhat larger than shown in figure 2. The stop watch was started once the field was reversed and stopped when the domain wall had traversed the area under examination. Provided we were within $50 \%$ of $V_{\mathrm{C}}$ so that the switching times, $\tau$, were fairly long, we found that $V \ln \tau$ (with $V$ in volts and $\tau$ in seconds) was constant to better than $7 \%$. For example, at $55.5{ }^{\circ} \mathrm{C}$, we found for $V=140,160$ and $180 \mathrm{~V}$, $\tau=33.1,27.5$ and $18 \mathrm{~s}$ respectively, giving an average $V \ln \tau=514 \pm 20[\mathrm{~V} \ln \mathrm{s}]$. In figure 3 we plot this parameter (with the observed spread as an error bar) as a function of temperature. Interestingly, we find a straight line which extrapolates to zero at $61.4{ }^{\circ} \mathrm{C}$ the $\mathrm{X}-\mathrm{G}^{*}$ transition temperature.

It is important to note that in order to make these observations, fairly well-oriented samples seem necessary. The change from state (a) to state (b) proceeds by wall motion in the layers (sketched in Fig. 1) and some degree of cooperativity may be necessary to effect this transition. When the domains are small (less than $50 \mu$, say) it is in fact difficult to observe any change in $\mathrm{X}$ with field.

The kind of wall motion we observed in $\mathrm{X}$ is different from that of $\mathrm{C}^{*}, \mathrm{I}^{*}$ and $\mathrm{G}^{* *}$ phases where wall motion proceeds perpendicular to the layers in what we have called the " soliton-switch " [9]. The electrooptic response in phases equipped with the helicoidal structure proceeds without a threshold field since in these phases, the allowed director orientation is infinitely degenerate on the surface of a cone. For example, once the field is turned off, a field oriented $\mathrm{C}^{*}$ phase will relax to some other configuration, as determined by surface forces, say. In X, we propose that the director can assume only a discrete set of orientations on the cone surface thus accounting for its threshold behaviour in a natural way, independent of the detailed crystallographic structure. Furthermore, figure 3 shows that $\tau=\exp \left\{C\left|T_{\mathrm{C}}-T\right| / V\right\}$ where $C$ is a positive constant where the free parameter, $T_{C}$, is identical, within the accuracy of our measurements, to the $\mathrm{G}^{\prime *} \rightleftarrows \mathrm{X}$ transition temperature. Qualitatively, one might interpret this relation as evidence of the existence of an activation energy to start director re-orientation. The factor $1 / V$ effectively reduces the activation energy.

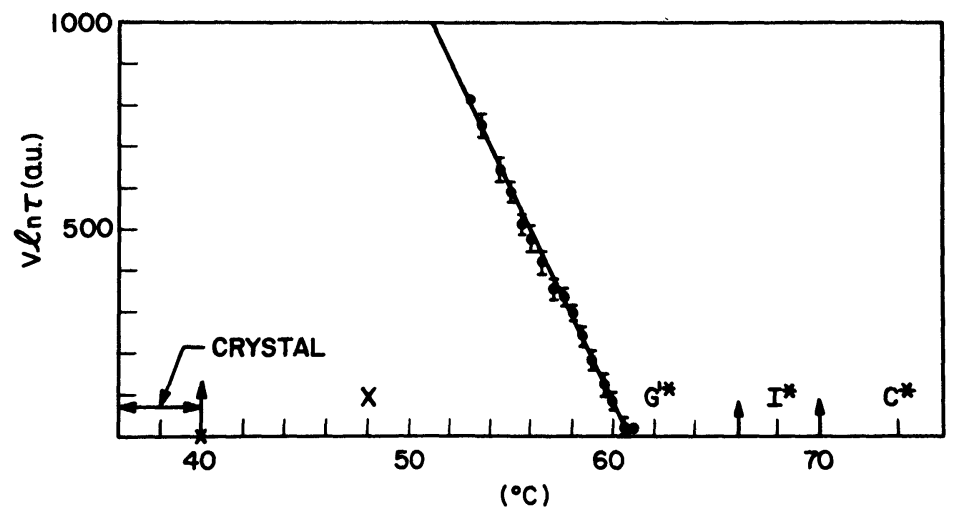

Fig. 3. - The product $V \cdot \ln \tau$ ( $V$ in volts, $\tau$ in seconds) plotted as a function of temperature. 
We believe the main interest in $\mathrm{X}$ is a fundamental one and, with the long intrinsic switching times of currently available materials, immediate utilization of $\mathrm{X}$ seems unlikely. However, coupled with a higher temperature, sub-micro-second switching but not bistable $\mathbf{C}^{*}$ phase, $\mathbf{X}$ could be useful for electrooptic storage rather like the smectic $A$ light valve [11]. In the $C^{*}$ phase of $8 \mathrm{SI}^{*}$, we observed switching times typically on the order of $10 \mu \mathrm{s}$ [12].

In concluding the discussion of the $\mathrm{X}$ phase of $8 \mathrm{SI}$, we mention that we have found $\mathrm{X}$ in other compounds : e.g., in C92M4(S-(+)-(4-2' methylbutyl) benzoate 4'-nonyloxybiphenyl) [13] and in the liquid crystalline phase of HOBACPC ( $\mathrm{p}$-phenyloxybenzilidene $\mathrm{p}^{\prime}$-amino-2 chloropropyl cinnamate) [14] which appears below [15] the smectic IV phase of Jain and Wahl [16]. In MORA12 (s-4-(6 methyl) octylresorcylidene-4'-dodecylaniline) below $24.5^{\circ} \mathrm{C}$ we have found an additional liquid crystal phase which shows bistability [17]. The fact that $X$ has been observed in a variety of compounds means that it is not likely to be the property of a unique in-plane structure, as we have indicated above.

\section{Acknowledgments.}

We thank John Goodby for providing us with $8 \mathrm{SI}^{*}$ and for valuable discussions. It is also a pleasure to thank Bill Brinkman for useful discussions.

\section{References}

[1] De Gennes, P. G., The Physics of Liquid Crystals (Clarendon Press, Oxford) 1974.

[2] Smectic I and F phases are distinguished by the relative orientation between a centred rectangular net in the plane of the layers and the tilt direction. It seems worth mentioning that I occurs at higher temperatures than the less ordered F-phase.

[3] Leadbetter, A. J., Gaughan, J., Kelly, B., Gray, G. W. and Goodby, J. W., J. Physique Colloq. 40 (1979) C3-178.

[4] Gane, P. A. C., Wrighton, P. G. and Leadbetter, A. J., Mol. Cryst. Liq. Cryst. 66 (1981) 247.

[5] Pindak, R., Sprenger, W. O., Bishop, D. J., Osheroff, D. D. and Goodby, J. W., Phys. Rev. Lett. 48 (1982) 173.

[6] Gane, P. A. C., Leadbetter, A. J., Benattar, J. J., Moussa, F. and Lambert, M., Phys. Rev. A 24 (1981) 2694.

[7] Meyer, R. B., Liébert, L., Strzelecki, L. and Keller, P., J. Physique Lett. 36 (1975) L-69.

[8] MeYer, R. B., Mol. Cryst. Liq. Cryst. 40 (1977) 33.

[9] Cladis, P. E., Brand, H. R. and Finn, P. L., Phys. Rev. A 28 (1983) 512.

[10] Kondo, K., Kobayashi, F., Fukuda, A. and Kuze, E., Jpn. J. Appl. Phys. 20 (1981) 1773.

[11] Kahn, F., Appl. Phys. Lett. 22 (1973) 111.

[12] BRAND, H. R. and Cladis, P. E. (unpublished).

[13] Brand, H. R., Cladis, P. E. and Goodby, J. W. (unpublished).

[14] Keller, P., Jugé, S., Líbert, L. and Strzelecki, L., C.R. Hebd. Séan. Acad. Sci. C 282 (1976) 639.

[15] Cladis, P. E., Brand, H. R. and Keller, P. (unpublished).

[16] JAIN, S. C. and WAHL, J., J. Physique Lett. 44 (1983) L-201. Independently of this work, these authors point out smectic IV $\left(\mathrm{G}^{\prime *}\right)$ may also be a bistable, ferro-electric liquid crystal like $\mathrm{X}$. We discuss this in ref. [15].

[17] Brand, H. R., Cladis, P. E., Otterholm, B. and Finn, P. L. (unpublished). 